\title{
VULNERABILIDADE DE IDOSOS ÀS QUEDAS SEGUIDAS DE FRATURA DE QUADRIL ${ }^{\mathrm{a}}$
}

\author{
Vulnerability of elderly falls followed for hip fracture \\ Vulnerabilidad de ancianos a las caídas seguidas de fractura de cadera
}

Lucimara Rocha $^{1}$

Rosângela Marion da Silva ${ }^{4}$
Maria de Lourdes Denardin Budó2

Juliana Petri Tavares ${ }^{5}$
Margrid Beuter ${ }^{3}$

\section{RESUMO}

Trata-se de uma pesquisa qualitativa, de natureza descritiva, realizada em uma unidade de clínica cirúrgica de um hospital universitário. 0 objetivo foi identificar os fatores de vulnerabilidade dos idosos às quedas seguidas de fratura de quadril. Os sujeitos foram treze cuidadores de idosos com fratura de quadril decorrentes de queda, hospitalizados no período de abril a novembro de 2008. Os instrumentos para coleta dos dados foram a consulta dos prontuários dos idosos e a entrevista semiestruturada. A análise se deu à luz do referencial de vulnerabilidade sendo os dados categorizados nas dimensões individual/ biológica, social e programática/institucional. Os resultados demonstraram que os idosos da pesquisa estão expostos de maneira interdependente às diferentes dimensões de vulnerabilidade às quedas. Dessa forma, conclui-se que o planejamento de ações dos profissionais de saúde devem contemplar igualmente aspectos individuais e coletivos dessa população, visando atenuar a incidência de quedas seguidas de fraturas do quadril.

Palavras-chave: Enfermagem. Saúde do Idoso. Vulnerabilidade em Saúde. Fraturas do Quadril. Hospitalização.

\begin{abstract}
The present study is a descriptive qualitative research that was developed in a clinical surgery unit of a university hospital. One aimed to identify elderly people vulnerability factors related to falls whose consequences were hip fractures. The subjects were thirteen caregivers of elderly patients with hip fractures after falls, hospitalized in the period from April to November 2008. The instruments of data collection were searching the promptuary of the elderly and structured interviews. Data were analyzed based on vulnerability referential and were categorized under individual/biological, social and programmatical/institutional dimensions. Thus, concluded that the planning of public health professionals should include the individual and collective aspects of this population, to mitigate the incidence of hip fractures after falls.
\end{abstract}

Keywords: Nursing. Health of the Elderly. Health Vulnerability. Hip Fractures. Hospitalization.

\section{Resumen}

Se trata de una investigación cualitativa, de naturaleza descriptiva, realizada en una unidad de clínica quirúrgica de un hospital universitario. El objetivo fue identificar los factores de vulnerabilidad de los ancianos a las caídas seguidas de fractura de cadera. Los sujetos fueron trece cuidadores de ancianos con fractura de cadera decurrentes de caída, hospitalizados en el período de abril a noviembre de 2008. Los instrumentos para la recolección de los datos fueron la consulta de los prontuarios de los ancianos y la entrevista semi-estructurada. El análisis se dio a la luz del referencial de vulnerabilidad siendo los datos categorizados en las dimensiones individual/biológica, social y programática/institucional. Los resultados demostraron que los ancianos de la investigación están expuestos de manera interdependiente a las diferentes dimensiones de vulnerabilidad a las caídas. De esa forma, se concluye que el planeamiento de acciones de los profesionales de salud debe contemplar igualmente aspectos individuales y colectivos de esa población, visando atenuar la incidencia de caídas seguidas de fracturas de cadera.

Palabras clave: Enfermería. Salud del Anciano. Vulnerabilidad en Salud. Fracturas de Cadera. Hospitalización.

\footnotetext{
'Enfermeira. Mestranda no Programa de Pós-Graduação em Enfermagem da Universidade Federal de Santa Maria (UFSM). Membro do Grupo de Pesquisa: Cuidado, Saúde e Enfermagem da UFSM. Santa Maria - RS. Brasil. E-mail: lucimarazz@hotmail.com.'2EEnfermeira. Doutora em Enfermagem. Professor Adjunto do Departamento de Enfermagem da UFSM. Membro do Grupo de Pesquisa: Cuidado, Saúde e Enfermagem da UFSM. Santa Maria - RS. Brasil. E-mail: lourdesdenardin@gmail.com,3.3nfermeira. Doutora em Enfermagem. Professor Adjunto do Departamento de Enfermagem da UFSM. Membro do Grupo de Pesquisa: Cuidado, Saúde e Enfermagem da UFSM. Santa Maria - RS. Brasil. E-mail: beuter@terra.com.br, ${ }^{4}$ Enfermeira do Hospital Universitário de Santa Maria, Mestre em Enfermagem. Membro do Grupo de Pesquisa: Trabalho, Saúde, Educação e Enfermagem da UFSM. Santa Maria - RS. Brasil. E-mail: cucasma@terra.com.br., ${ }^{5}$ Enfermeira. Mestranda no Programa de Pós-Graduação em Enfermagem da UFSM. Membro do Grupo de Pesquisa: Trabalho, Saúde, Educação e Enfermagem da UFSM. Santa Maria - RS. Brasil. E-mail: julianapetritavares@yahoo.com.br.
} 


\section{INTRODUCÃO}

As quedas em idosos são consideradas um importante problema de saúde pública, em função de sua incidência, complicações e custos ao sistema de saúde. Esses agravos ocasionam perdas da autonomia e da independência do idoso por estarem diretamente relacionados a ocorrência de fraturas, especialmente as de quadril.

No Brasil, em quatro anos (2005 a 2008), ocorreu o aumento do número de internações em $8 \%$ por fratura quadril em idosos. Em 2008, esse tipo de fratura foi responsável por 32.908 internações hospitalares na rede do Sistema Único de Saúde, com um custo total de $\mathrm{R} \$ 58,6$ milhões. ${ }^{1}$

A fratura de quadril está também entre as causas relevantes de morbidade e mortalidade dos idosos. Entre as causas externas, as quedas são responsáveis por $24 \%$ das mortes, enquanto correspondem a $6 \%$ no restante da população. Cerca de $30 \%$ dos idosos sofrem quedas a cada ano, sendo que essa taxa aumenta para $40 \%$ entre os idosos com mais de 80 anos. Entre os idosos até 75 anos, as mulheres tendem a cair mais que os homens e, a partir dessa idade, as frequências se igualam.

Tendo em vista o cenário em questão, percebe-se a importância em discutir medidas de atenção à saúde do idoso, que ultrapassem o plano biológico, a fim de contemplar outras dimensões como as sociais e institucionais. Partindo desse pensamento, empregou-se o conceito de vulnerabilidade de Ayres. $^{2}$

A utilização do conceito de vulnerabilidade envolve os agravos e seus impactos em totalidades dinâmicas formadas por aspectos que vão de suscetibilidades orgânicas à forma de estruturação de programas de saúde, passando por aspectos comportamentais, culturais, econômicos e políticos, ${ }^{2}$ possibilitando intervir sobre outros agravos, como as quedas de idosos seguidas de fratura de quadril, identificando suas razões determinantes.

A utilização do termo vulnerabilidade tem sido crescente no campo da saúde coletiva na enfermagem. Primeiramente, o conceito de vulnerabilidade foi empregado na área dos Direitos Humanos para designar indivíduos ou grupos fragilizados jurídica ou politicamente, para promoção, proteção ou garantia de seus direitos de cidadania. ${ }^{2}$ Este conceito foi incorporado à área da saúde com os estudos sobre a AIDS realizados na Escola de Saúde Pública de Harvard, Estados Unidos, no ano de 1993. No que tange ao processo de saúde-doença, a vulnerabilidade é um indicador da iniquidade e desigualdade social. ${ }^{3}$

No presente estudo, a vulnerabilidade descreve a possibilidade de exposição das pessoas ao adoecimento, como resultante de um conjunto de aspectos que, embora se refiram imediatamente ao indivíduo, o recoloca na perspectiva da dupla-face, ou seja, o indivíduo e sua relação com o coletivo. ${ }^{4}$
Ayres $^{2}$ define três planos interdependentes de determinação de maior ou menor vulnerabilidade dos indivíduos e coletividades aos agravos de saúde: o comportamento pessoal ou vulnerabilidade individual/biológica; o contexto social ou vulnerabilidade social; e as ações governamentais para enfrentar o agravo ou vulnerabilidade programática/ institucional.

0 plano individual diz respeito ao grau e à qualidade da informação que os indivíduos dispõem sobre o problema, à capacidade de processar essas informações e incorporá-las ao seu cotidiano de preocupações e às possibilidades de transformar essas preocupações em práticas.?

0 plano social considera que a aquisição de informações, as possibilidades de processá-las e incorporá-las não dependem apenas dos indivíduos, mas de aspectos como acesso aos meios de comunicação, grau de escolaridade, disponibilidade de recursos materiais, poder de influenciar decisões políticas e possibilidade de enfrentar barreiras culturais. ${ }^{2}$

No plano programático, é fundamental a existência de esforços governamentais, para que os recursos sociais necessários aos indivíduos sejam disponibilizados de modo efetivo e democrático a fim de não se exporem aos danos. ${ }^{2}$

Tendo em vista a realidade exposta e a fundamentação no referencial de vulnerabilidade, buscou-se neste estudo responder à seguinte questão norteadora: quais os fatores que influenciam a vulnerabilidade dos idosos às quedas seguidas de fratura de quadril? Visando responder essa questão, o objetivo foi identificar os fatores de vulnerabilidade dos idosos às quedas seguidas de fratura de quadril.

\section{PERCURSO METODOLÓGICO}

Trata-se de uma pesquisa qualitativa, de natureza descritiva, realizada na unidade de Clínica Cirúrgica de um Hospital Universitário no interior do Rio Grande do Sul. Essa unidade hospitalar foi escolhida uma vez que os idosos que são submetidos à correção de fratura ou artroplastia de quadril aí permanecem internados.

Os sujeitos da pesquisa foram os cuidadores de idosos hospitalizados que atenderam ao seguinte critério de inclusão: ser cuidador familiar de idoso (pessoa com idade igual ou superior a 60 anos) internado devido à fratura de quadril motivada por queda.

A coleta de dados ocorreu de abril a novembro de 2008 em dois momentos. No primeiro momento, foi realizada uma busca na unidade de Clínica Cirúrgica para identificar os idosos submetidos aos cuidados da especialidade traumatológica seguida da consulta aos seus prontuários. No segundo momento, foi utilizada a entrevista semiestruturada com os cuidadores de idosos que atenderam ao critério de inclusão e aceitaram participar do estudo.

Os cuidadores foram entrevistados nos locais de internação dos idosos, sendo explicado o objetivo da pesquisa, 
bem como o caráter voluntário de participação com garantia de anonimato. 0 consentimento do cuidador ocorreu mediante a assinatura do Termo de Consentimento Livre e Esclarecido. A entrevista foi registrada por meio de gravação em áudio, previamente autorizada.

As entrevistas foram transcritas na íntegra, mantendose o sentido original do que foi dito. Esses dados, bem como os obtidos na consulta aos prontuários dos idosos, foram identificados pela letra maiúscula $C$, de cuidador, seguido do número correspondente à ordem da coleta das informações (C1, C2 ...), a fim de preservar a identidade dos participantes da pesquisa.

A pesquisa foi aprovada pelo Comitê de Ética em Pesquisa da Universidade Federal de Santa Maria sob número do Certificado de Apresentação para Apreciação Ética (CAAE) 0033.0.243.000-08 de 11 de março de 2008, atendendo, assim, aos requisitos da Resolução 196/96 do Conselho Nacional de Saúde que trata da questão ética em pesquisas envolvendo seres humanos. ${ }^{5}$

Foram entrevistados treze cuidadores que acompanhavam os idosos internados que haviam sofrido fratura de quadril ocasionado por queda. A delimitação do número de entrevistas aconteceu quando se observou que os dados coletados já respondiam ao objetivo da pesquisa, percebendose a saturação destes; assim, o número de entrevistados foi considerado satisfatório. ${ }^{6}$

As entrevistas foram transcritas, e foi realizada a leitura do material a fim de identificar o conteúdo manifesto, agrupando-se os fragmentos que possuíam semelhança semântica nos diferentes depoimentos, considerando as dimensões propostas pelo construto conceitual da vulnerabilidade. Os elementos que contribuíram para a vulnerabilidade dos idosos às quedas seguidas de fratura de quadril foram identificados conforme os planos individual/ biológico, social e programático/institucional.

\section{ANÁLISE E DISCUSSÃO DE RESULTADOS}

A partir da análise, os dados foram organizados considerando os aspectos relacionados à vulnerabilidade biológica/individual, vulnerabilidade social e vulnerabilidade programática/institucional aos quais estão expostos os idosos da pesquisa.

\section{Vulnerabilidade biológica/individual de idosos às quedas seguidas de fratura de quadril}

A vulnerabilidade biológica/individual representa o que a pessoa é, pensa, faz e quer, e o que, ao mesmo tempo, a expõe ou não à aquisição de um agravo à saúde. ${ }^{7}$ Assim, no componente individual de vulnerabilidade, incluem-se, entre outros, sexo, raça, idade e situação de saúde atual.

Dos treze idosos, doze pertenciam ao sexo feminino e um ao sexo masculino, dez eram de raça branca, dois eram negros e um, pardo. 0 fato de encontrarmos a maioria de idosas internadas por quedas vem ao encontro de estudo, ${ }^{8}$ que sugere como causas desse evento a maior fragilidade das mulheres em relação aos homens. Nesse trabalho foram investigados dados referentes à quantidade de massa óssea e força muscular, assim como a maior prevalência de doenças crônicas, supondo, ainda, que o fato possa estar relacionado às atividades domésticas exercidas predominantemente pelas mulheres.

Quanto à raça branca, um estudo epidemiológico acerca das fraturas do fêmur proximal ${ }^{9}$ aponta que essa raça encontrase mais propensa às fraturas consequentes às quedas, pois apresenta maior tendência de desenvolver osteoporose, enquanto a raça negra apresenta maior massa óssea, o que reduz a possibilidade de fraturas.

Com relação à faixa etária, os dados evidenciaram que três dos idosos tinham entre 90 e 99 anos; cinco, entre 80 e 89 anos; três, entre 70 e 79 anos; e dois, entre 60 e 69 anos. Desta forma, verifica-se que o número de idosos que sofrem queda seguida de fratura é maior conforme a evolução da idade. Assim, o aumento da expectativa de vida da população apresenta como uma de suas consequências uma maior tendência à exposição dos indivíduos a esse tipo de agravo.

Quanto à situação de saúde atual, os idosos (12) apresentavam outros problemas de saúde, que podem ser associados à fratura do quadril, como as alterações osteomusculares, (artrose hemiplegia de membros e osteoporose), alterações cardiovasculares, neurológicas, endócrinas, psiquiátricas e neoplásicas. Além dessas alterações, constatou-se que os idosos apresentavam dificuldades visuais e auditivas. $^{8}$

Embora essas doenças não sejam exclusivas das faixas etárias mais avançadas, elas afetam o estado atual de saúde dos idosos bem como o seu agravamento, porém, não são consequências inevitáveis do processo de envelhecimento. A prevenção é efetiva em qualquer nível, independente da idade do indivíduo. ${ }^{10}$

Quanto à questão da situação atual de saúde, considerando-se o uso de medicamentos, todos os idosos faziam uso contínuo de algum fármaco, utilizando de um a nove tipos de medicamentos concomitantemente. Os medicamentos mais consumidos pelos idosos foram os antihipertensivos, os ansiolíticos e os antidepressivos/indutores do sono. Um estudo ${ }^{11}$ considera que 0 uso destes medicamentos tem como efeitos colaterais a hipotensão, hipoglicemia, síncopes, astenia muscular, entre outras, o que pode facilitar os eventos de queda dos idosos.

Ao tratar da vulnerabilidade individual dos idosos às quedas e à determinação do estado de saúde atual, os cuidadores foram questionados quanto à ocorrência de queda anterior à que originou a hospitalização atual do idoso. Onze dos entrevistados responderam a questão afirmativamente, como ilustram as falas a seguir: 
Caía seguido [...] ela tropicava e sempre caia de costas, agora ela caiu de lado. $\left(C_{3}\right)$

Volta e meia ela cai, tira o couro [pele] do joelho, não foi o primeiro tombo dela. Ela já caiu diversas vezes, tombinhos que não levam a nada, leves. $\left(C_{8}\right)$

Dos idosos que já haviam caído anteriormente, seis sofreram fraturas. Segundo os cuidadores, as quedas anteriores estavam relacionadas ao estado de fraqueza e tonturas dos idosos, como mostram as falas:

Muitas quedas, com muitas fraturas. A do fêmur, a do braço, quebrou as costela umas duas vezes, tudo por queda. Ela não conseguia se firmar mais, sentia dificuldades [...] ela acabou caindo no banheiro. $\left(C_{4}\right)$

Ela estava fazendo suco e se virou, acho que tonteou a cabeça e caiu. $\left(C_{10}\right)$

Fundamentado nos relatos, é possível identificar elementos referentes à vulnerabilidade individual/biológica dos idosos que os predispõem às quedas recorrentes. Tal agravo tende a levar ao declínio funcional e encaminha o idoso ao desenvolvimento de incapacidades, tornando-o mais frágil. 0 declínio da capacidade funcional geralmente conduz a pessoa idosa à limitação ou perda da capacidade de desempenhar de forma independente suas atividades de vida diária, atingindo outros planos de vulnerabilidade. ${ }^{7}$

\section{Vulnerabilidade social de idosos às quedas seguidas de fratura de quadril}

A vulnerabilidade social é atribuída ao modo de "obtenção de informações [...], como acesso aos meios de comunicação, escolaridade, disponibilidade de recursos materiais e possibilidade de enfrentar barreiras culturais [...]" 2: 123. Dessa forma, a vulnerabilidade social do idoso é decorrente de uma diversidade de circunstâncias com as quais essas pessoas convivem cotidianamente.

Um dos pontos a investigar no que tange à vulnerabilidade social dos idosos foi identificar com quem estes residiam. Nesse sentido, foi verificado que os idosos residiam com o cônjuge, com os filhos, sozinhos, com outros familiares e em instituição de longa permanência. A maioria dos idosos residia com o seu núcleo familiar. Os idosos que moravam com o cônjuge tinham os filhos vivendo nas proximidades, denotando uma vivência intergeracional nas famílias nas quais membros de diferentes gerações convivem próximos, caracterizando a rede de apoio ao idoso. A família é a mais direta fonte de apoio informal aos idosos, o que se verifica em situações de corresidência ou não. Os membros de uma família são solidários na busca do bem-estar coletivo, constituindo um espaço de conflito cooperativo, em que se cruzam as diferenças por gênero e intergeracionais. ${ }^{12}$

Em relação à escolaridade, onze idosos haviam cursado o ensino fundamental incompleto e um, o ensino médio incompleto. Acerca das atividades que desenvolveram ao longo da vida, cinco idosos trabalharam na agricultura, cinco exerciam atividades no setor de serviços nas áreas de comércio e alimentação e três exerciam atividades no lar. Todos os idosos eram aposentados, seja por idade ou por meio da aposentadoria rural, com renda mensal igual a um salário mínimo. A pouca escolaridade acompanhada de baixa renda pode contribuir para a vulnerabilidade social dos idosos devido à precária remuneração previdenciária diante dos recursos necessários para manutenção de uma condição de vida saudável.

Em relação ao estado civil, sete eram casados; quatro, viúvos; um, separado; e um, solteiro. Dos viúvos, a totalidade era de mulheres. Esse dado pode ser explicado pelo fato de as mulheres, em geral, terem uma expectativa de vida maior que a dos homens. ${ }^{1}$

Quanto ao número de filhos, um não os tinha, três tinham um filho, sete tinham menos de cinco filhos e dois tinham mais que dez filhos. Dessa forma, a maioria dos idosos não tinha família numerosa, indo ao encontro de dados divulgados ${ }^{13}$ que demonstram que a família brasileira está cada vez menor, passando da média de 3,6 pessoas, em 1996, para 3,2, em 2006.

Sobre as situações ambientais que podem expor os idosos às quedas e com as quais essas pessoas têm que lidar em suas casas cotidianamente, as relatadas foram os degraus na soleira das portas, tapetes soltos, escadas sem corrimão, objetos guardados em locais altos, piso escorregadio, banheiro com piso escorregadio, calçados inadequados, cadeiras/camas muito altas ou muito baixas, móveis instáveis e deslizantes e iluminação deficiente.

Nesta investigação, os dados permitiram identificar que, em dez ocasiões, as quedas aconteceram predominantemente no ambiente domiciliar, havendo prevalência quanto ao cômodo da residência pela sala - com quatro ocorrências. Em relação ao período em que os idosos caíram, constatou-se que o horário de maior ocorrência de quedas foi durante o dia, principalmente na tarde e início da noite, contrariando o conhecimento popular que atribui como horário de maior risco para quedas o período da noite.

Sobre a situação e causa da queda que originou a fratura do quadril e que podem ser relacionadas à vulnerabilidade social dos idosos, diferentes situações foram relatadas: uso de cadeiras muito baixas, escorregões em superfícies úmidas, utilização de calçado inadequado, atrapalhar-se com degraus da escada e tapetes, como mostram os relatos:

[...] foi assim, ela estava sentadinha e foi para levantar da cadeira de praia caiu e fraturou. (C5) 
Vulnerabilidades de idosos às quedas com fraturas

Rocha L, Budó MLD, Beuter M, Silva RM, Tavares JP

[...] ela me disse que tropicou com o chinelo $[\ldots] \cdot(C 3)$

[...] dessa vez ela foi levantar tinha água, ou tinha um xixi, porque eles levam o pinico de noite para 0 quarto, ela foi levantar da cama e foi se apoiar na janela, resvalou e caiu. (C8)

[...] quando ela vinha voltando, acho que ela errou o degrau, foi na escada... (C6)

Ela estava sentada na cadeira, daí quis colocar a cadeira para fora para sentar ver a rua e daí o pé pegou numa pontinha do tapete, mas numa pontinha, quando viu caiu. (C11)

Os dados coletados, reforçados pelos relatos acima, apontam que oito das quedas, que deram origem à fratura do quadril nos idosos do estudo, apresentaram como causa as condições ambientais durante a realização de atividades do dia-a-dia. Portanto, diante das condiç̃es ambientais que tornam os idosos mais vulneráveis às quedas, os relatos dos cuidadores evidenciaram como forma de cuidado a retirada dos idosos de seus domicílios como medida preventiva de novas quedas e fraturas, como sinalizam as falas a seguir:

[...] agora vou ter que levá-los lá para casa, não dá mais agora, a mãe não pode ir mais para a roça. (C13)

Meu vô faleceu faz uns treze anos, até aí eles moravam juntos, depois ela ficou morando sozinha até $o$ ano passado quando ela caiu e teve a fratura das costelas, daí colocamos ela numa casa de repouso [...] não tinha mais como morar sozinha, não tinha condições com essa idade toda. (C4)

Os riscos de quedas e acidentes domésticos são maiores quando a habitação não está adaptada às necessidades dos idosos. A habitação representa a defesa do indivíduo das condições do meio ambiente em qualquer idade. A vida solitária de idosos ou de viúvas é pouco aconselhável, do ponto de vista físico e psíquico. Uma alternativa das famílias a esta realidade tem sido as residências geriátricas. ${ }^{14}$

A maioria das quedas sofridas pelos idosos, de acordo com os cuidadores, foi relacionada a uma condição de inevitabilidade, identificando-se um alto grau de banalização do evento, apesar de a maioria dos idosos terem sofrido quedas anteriores e algumas delas com fraturas, como demonstram os depoimentos a seguir:

Foi uma fatalidade, não tinha o que fazer. (C7)
Esc Anna Nery (impr.)2010 out-dez; 14 (4):690-696

Caia seguido, às vezes no meio das pedras, perto de casa, nunca se machucou [...]. As duas irmãs dela também caíram, uma quebrou o mesmo lado que ela e a outra quebrou mais em cima. (C3)

Ela caiu vários tombos, tombos até bem mais fortes que esse [...] só que o médico disse que ela caiu com uma perninha por cima da outra, e nos outros tombos ela caiu atirada, ela batia a cabeça, parecia que ia quebrar. (C5)

É possível perceber que as questões que participam da vulnerabilidade social dos idosos do estudo às quedas seguidas de fratura de quadril estão permeadas pelo contexto cultural em que os idosos e seus cuidadores estão inseridos. De um modo geral, a formação cultural influencia muitos aspectos da vida das pessoas, podendo ocasionar reflexo na saúde e na assistência. ${ }^{15}$ Banalizar o evento queda, considerando-o inerente ao processo de envelhecimento, é apenas um exemplo do estereótipo de idoso presente na sociedade.

Um dos questionamentos do estudo foi o de como impedir que os idosos sofram quedas evitando as fraturas. A aplicação de medidas de prevenção de quedas de idosos teve entendimentos divergentes. As falas a seguir exemplificam duas situações verificadas:

Eu lá em casa já sei o que vou fazer... vou desmanchar aqueles degraus, vou fazer uma rampa, daí colocamos dois corrimãos, um de cada lado... (C6)

Isso eu não posso te falar, não sei, sou da roça. (C13)

Estas falas evidenciam a influência da formação cultural e social como fatores determinantes para a percepção e a tomada de ações que evitem a ocorrência de quedas de idosos. Esta realidade é preocupante, pois as quedas representam significativo agravo à saúde, afetando de maneira desastrosa a vida dos idosos e de suas famílias.

Desta forma, percebe-se a importância do plano social no contexto da busca de informações, da capacidade de empoderamento que não dependem apenas dos indivíduos, mas das relações sociais, econômicas, culturais e políticas da realidade vivenciada pelo idoso e de sua família.

Vulnerabilidade programática/institucional de idosos às quedas seguidas de fratura de quadril

A vulnerabilidade programática abrange o grau e a qualidade de compromisso, recursos, gerência e monitoramento de programas nacionais, regionais ou locais de prevenção e cuidados. Esse componente da vulnerabilidade orienta ações educativas para além do 
caráter normativo e centrado no objeto, repensando ações em saúde como encontros de sujeitos. ${ }^{2}$

0 pouco acesso à informação sobre medidas de tratamento e recuperação da saúde dos idosos, como a ausência de ações copartícipes entre profissionais de saúde e cuidadoras, caracterizou-se como componente da vulnerabilidade programática dos idosos às quedas seguidas de fratura de quadril. A maioria dos cuidadores relatou não terem sido informados sobre os cuidados necessários ao tratamento, como exemplifica a fala:

Eu não sei para que serve [tração]. Só me disseram. Acho que foi até uma enfermeira, que me falou, que serve para colocar o osso da perna um pouco no lugar... Para ajudar a ir para o lugar. (C2)

0 desconhecimento do tratamento pode trazer riscos à saúde dos idosos, especialmente nos casos de reincidência de quedas. Os profissionais que assistem os pacientes devem fornecer informações aos cuidadores no preparo para a alta hospitalar. Pois, diante da falta de informações do cuidador e das demandas do cuidado, impostas pelo familiar doente, ele assume a responsabilidade de cuidar mesmo sem ter o adequado preparo. ${ }^{16}$

Em relação à participação dos cuidadores na terapêutica proposta para o tratamento, ocorrem situações de exclusão como a relatada na fala seguinte:

Eu queria falar com o médico que fez a cirurgia, para ver o que ele me diz, porque ele manipulou ela, mas não tem Cristo de falar com esse homem. Eu vou ter que ir no consultório dele, pagar uma consulta como se fosse para mim para falar dela. Eu não vi o homem, eu não conheço ele [...]. Não tenho nem noção do tratamento que ela fez [...]. Eu nunca vi... não conversar com o médico. 0 médico não vir conversar com o familiar. Acho que eu vou ter que ir na ouvidoria fazer queixa. É demais o descaso, ter que sair aí correndo atrás dele. (C4)

A falta de interação entre os profissionais de saúde e os cuidadores dos idosos é um fator que dificulta 0 acesso às medidas de promoção à saúde, 0 que favorece a vulnerabilidade dos idosos. Esse problema exige atenção especial do enfermeiro no sentido de adotar modelos assistenciais que envolvam estratégias educativas para a promoção da saúde do idoso e sua qualidade de vida, estimulando sua participação e autocuidado. ${ }^{17}$

\section{CONSIDERAÇÕES FINAIS}

0 estudo possibilitou a aplicação do conceito de vulnerabilidade nos eventos de queda seguida de fratura de quadril em idosos hospitalizados, considerando o problema de uma forma ampla. Buscou-se, a partir do referencial de vulnerabilidade, descrever esse agravo não somente a partir do determinante biológico, visto como característico do processo de envelhecimento, mas associar as condições sociais e programáticas que condicionam a ocorrência de quedas seguidas de fratura do quadril em idosos.

Os dados apresentados demonstram que os idosos do estudo têm elevada vulnerabilidade individual/biológica, social e programática/institucional de sofrerem quedas seguidas de fratura de quadril. É importante, dessa forma, o planejamento e a implementação de programas de promoção à saúde do idoso, considerando o problema em sua integralidade, para que possamos efetivamente reduzir os índices de morbimortalidade por quedas em idosos no Brasil.

Portanto entende-se como necessária a instrumentalização dos profissionais de saúde, principalmente os profissionais da enfermagem, no cuidado à saúde do idoso. É importante considerar o preparo e o compromisso profissional para um cuidado integral e contextualizado ao idoso, reconhecendo aspectos individuais e coletivos dessa população, visando à promoção de sua saúde.

0 fato de este estudo ter como informantes os cuidadores de idosos internados vítimas de quedas seguidas de fratura de quadril pode ser considerado um fator limitante, uma vez que os dados coletados retratam a vivência dos idosos sob o olhar dos seus cuidadores.

Sugere-se que investigações posteriores contemplem esse assunto com uma população mais abrangente, em outras regiões do país e em outros níveis de atenção à saúde, tendo em vista a relevância da temática.

\section{REFERÊNCIAS}

1 Ministério da Saúde (BR). Internações por fratura de fêmur crescem 8\% em quatro anos. [notícia da Internet] Brasília (DF); 2009 [citado 2009 ago 20] Disponível em: http://portal.saude.gov.br/portal/aplicacoes/noticias/ default.cfm?pg=dspDetalheNoticia\&id_area=124\&CO_NOTICIA=10063

2 Ayres JRCM, França Júnior I, Calazans GJ, Saletti Filho HC. 0 conceito de vulnerabilidade e as práticas de saúde: novas perspectivas e desafios. In: Czeresnia D, Freitas CM, organizadores. Promoção da saúde: conceitos, reflexões, tendências. Rio de Janeiro(RJ): Fiocruz; 2003. p. 117-39.

3 Ayres JRCM.Vulnerabilidade e AIDS: para uma resposta social à epidemia. Secretaria de Estado da Saúde de São Paulo/Programa de DST/AIDS. Bol Epidemiol. 1997; 15(3): 2-4. 
4 Muñoz SAl, Bertolozzi MR. Pode o conceito de vulnerabilidade apoiar a construção do conhecimento em saúde coletiva? Cienc Saude Colet. 2007 abr; 12(2): 319-24.

5 Ministério da Saúde (BR). Conselho Nacional de Saúde. Resolução nº 196, de 10 de outubro de 1996. Diretrizes e normas regulamentadoras de pesquisas envolvendo seres humanos. Inf Epidemiol SUS. 1996; 5 (2 supl 3): 13-41.

6 Minayo MCS. 0 desafio do conhecimento: pesquisa qualitativa em saúde. $10^{a}$ ed. São Paulo (SP): Hucitec; 2007.

7 Paz AA, Santos BRL, Eidt OR. Vulnerabilidade e envelhecimento no contexto da saúde. Acta Paul Enferm. 2006; 19 (32): 338-42.

8 Perracini MC, Ramos LR. Fatores associados a quedas em uma coorte de idosos residentes na comunidade. Rev Saude Publica. 2002; 36(6): 709-16.

9 Rocha MA, Carvalho WS, Zanqueta C, Lemos SC. Estudo epidemiológico retrospectivo das fraturas do fêmur proximal tratados no Hospital Escola da Faculdade de Medicina do Triângulo Mineiro. Rev Bras Ortop. 2001; 36(08): 311-16.

10 Veras R. Envelhecimento populacional contemporâneo: demandas, desafios e inovações. Rev Saude Publica. 2009; 43 (3): 548-54.

11 Ribeiro AP. Repercussões das quedas na qualidade de vida de mulheres idosas. [dissertação] Rio de Janeiro (RJ): Instituto Fernandes Figueira, Fundação Oswaldo Cruz; 2006.
12 Camarano AA. Os novos idosos brasileiros muito além dos 60 ? A família como locus de apoio e de trocas intergeracionais. Rio de Janeiro (RJ): Ipea; 2004.

13 Instituto Brasileiro de Geografia e Estatística- IBGE. Pesquisa Nacional por Amostra de Domicílios/PNAD [on-line] 2007; [citado 2009 ago 15] Disponível em http://www.ibge.gov.br.

14 Moragas MR. Gerontologia social, envelhecimento e qualidade de vida. São Paulo (SP): Paulinas; 1997.

15 Helman CG. Abrangência da antropologia médica. In: Cultura, saúde \& doença. $4^{\mathrm{a}}$ ed. Porto Alegre (RS): Artes Médicas; 2003.

16 Brondani CM. Desafio de cuidadores familiares no contexto da internação domiciliar [dissertação]. Santa Maria (RS): Universidade Federal de Santa Maria; 2008.

17 Monteiro CFS, Luz MHA. Educação em saúde e mulheres idosas: promoção de conquistas políticas, sociais e em saúde. Esc Anna Nery 2006 dez; 10(3): 456-61.

\section{NOTA}

a Este artigo é um recorte de um trabalho de conclusão de curso apresentado em julho de 2008 ao Curso de Graduação em Enfermagem da UFSM, Santa Maria, RS 